\title{
Atrazine dissipation in a biobed system inoculated with immobilized white-rot fungi
}

\author{
Sebastian Elgueta ${ }^{a}$, Cledir Santos ${ }^{\mathrm{a}}$, Nelson Lima ${ }^{\mathrm{b}}$ and Maria Cristina Diez \\ aDepartment of Chemical Sciences and Natural Resources, Faculty of Engineering and Sciences, Universidad de La \\ Frontera, Temuco, Chile; ${ }^{b} \mathrm{CEB}$-Centre of Biological Engineering, Micoteca da Universidade do Minho, University of \\ Minho, Campus of Gualtar, Braga, Portugal; 'Department of Chemical Engineering, Faculty of Engineering and \\ Sciences, Universidad de La Frontera, Temuco, Chile
}

\begin{abstract}
Due to environmental concerns about accumulation of the herbicide atrazine in food products and water reservoirs, there is a need to develop safe and economical methods for its dissipation. The main aim of this study was to evaluate atrazine dissipation in a biobed system inoculated with immobilized white-rot fungi in a pelletized support (PS). All fungal isolates evaluated were efficient in colonizing the surface and inner parts of the PS, with no differences observed in the colonization over the assay. The highest atrazine dissipation (93\%) was observed for Stereum hirsutum Ru-104 after 60 days of incubation. In contrast, the lowest atrazine dissipation (78\%) was observed for the non-inoculated biobed over an identical period. Trametes versicolor HL01 was involved in the highest phenoloxidase activity. This activity indicates that inoculated and non-inoculated biobeds were biologically active over the incubation period. A high respiration rate in the biobed was observed when it was inoculated with S. hirsutum Ru-104. Inoculation of fungi on PS systems may be a coadjutant in the increase of fungal efficiency in enzymatic production.
\end{abstract}

\section{ARTICLE HISTORY}

Received 31 July 2015

Accepted 5 February 2016

\section{KEYWORDS}

White-rot fungi; biobed; atrazine; pelletized support; biological activity

\section{Introduction}

The widespread contamination of soil with chlorinated aromatic herbicides is an important environmental and societal concern. When it can have a negative influence on the quality of soil, it can also become a potential threat to human health (Bastos \& Magan 2009). Atrazine is a chlorinated aromatic herbicide that is used worldwide for the control of broad-leaved weed in agricultural production (Ralebitso et al. 2002), as well as in urban and recreational green areas. Its inadequate management on farms, especially related to its handling, appears to be the most important source of contamination. Furthermore, spillage can often occur, particularly during the filling of spraying equipment, which is described as a point source of contamination. Due to the environmental concerns associated with the accumulation of atrazine in food products and water reservoirs, there is a need to develop safe and economical methods for the dissipation of this pesticide (Zhang \& Qiao 2002).

Biobed is a biopurification system representing a cost-effective technology for dissipation of pesticides. It is composed of straw, peat and soil that absorbs and facilitates pesticide dissipation. Due to biobed microbial activity, especially the enzymatic activity of white-rot fungi (Castillo et al. 2008), pesticide dissipation can occur. Although the precise role of enzymes in pesticide dissipation 
by white-rot fungi has not yet been completely established, evidence suggests that a complex of enzymes, which includes lignin peroxidases (LiP, EC 1.11.1.14), manganese peroxidases (MnP, EC 1.11.1.13) and laccases (LCC, EC 1.10.3.2), is responsible at least in part for the degradative capability of biobeds (Tortella et al. 2008).

Rodríguez-Rodríguez et al. (2013) described the importance of white-rot fungi in pesticide dissipation through its bioaugmentation in a biopurification system. In addition, various strains of Phanerochaete chrysosporium, Pleurotus ostreatus and Trametes versicolor have shown marked ability to biodegrade different classes of pesticide (Mougin et al. 1997; Castillo et al. 2001).

The application of white-rot fungi in the biodegradation process has been used mainly through the addition of pre-grown fungal cultures on lignocellulosic supports as a pre-adaption medium. It can improve the fungal ability to survive in the presence of other soil microorganisms (D'Annibale et al. 2006). Several types of lignocellulosic support have been used to introduce pre-inoculated white-rot fungi into the soil (Leštan et al. 1996; Walter et al. 2005). This practice can facilitate the access and consumption of specific nutrients by fungi, leading to cellular growth (Walter et al. 2005; Rubilar et al. 2011). Immobilized fungi have potential to improve atrazine dissipation in a biobed system. The main aim of the present study was to evaluate atrazine dissipation in a biobed system with immobilized white-rot fungi.

\section{Materials and methods}

\section{Fungal strains}

Stereum hirsutum Ru-104, Inonotus sp. SP2 and Trametes versicolor HL01 were isolated from dead wood in Rucamanque Park in southern Chile. Strains were preserved and supplied by the Environmental Biotechnology Laboratory, Universidad de La Frontera, Chile. Fungi were transferred from slant cultures to glucose malt extract agar plates (GMEA, $10 \mathrm{~g} \mathrm{~L}^{-1}$ glucose, $30 \mathrm{~g} \mathrm{~L}^{-1}$ malt extract, $15 \mathrm{~g} \mathrm{~L}^{-1}$ agar, $\mathrm{pH} \mathrm{5.2)} \mathrm{and} \mathrm{incubated} \mathrm{at} 25 \pm 1^{\circ} \mathrm{C}$ for 7 days.

\section{Pelletized support and fungal immobilization}

A support including $74 \%$ sawdust, $6 \%$ starch, $2 \%$ corn meal, $15 \%$ flaxseed and $3 \%$ lignosulphonate was prepared for fungal immobilization (patent requested 2013-1395, Chile). In order to obtain a pelletized support (PS) of $8 \mathrm{~mm}$, ingredients were mixed, moistened to approximately 10\% humidity with distilled water and pressed using a pellet mill (ZLSP300B R-Type). Ten grams of PS were transferred to polypropylene bags and moistened with $4 \mathrm{~mL}$ of GMEA. Fungal immobilization was produced by inoculation with five plugs of active mycelium of $S$. hirsutum Ru-104, Inonotus sp. SP2 or T. versicolor HL01 previously grown on GMEA plates. Finally, bags containing inoculated PS were incubated at $25 \pm 1^{\circ} \mathrm{C}$ for 30 days.

\section{Scanning electron microscopy}

Photomicrographs were taken using a scanning electron microscope (SEM, Leica/Cambridge Instrument S360, Cambridge, UK). Samples from the surface and center of the PS were prepared by cutting the immobilized fungus into small pieces. For all formulations, samples were cut to the same length $(2 \mathrm{~cm})$ using a sterilized knife. Samples were fixed with $2.5 \%$ glutaraldehyde for $1.5 \mathrm{~h}$ at $4^{\circ} \mathrm{C}$, followed by $0.1 \mathrm{M}$ cacodylate salt buffer $\mathrm{pH} 7.0$ for $30 \mathrm{~min}$, then post-fixed with $1 \%$ osmium tetroxide, dehydrated with acetone, dried and metalized with gold. 


\section{Biomixture formulation and biobed system establishment}

The biomixture was prepared as previously described (Urrutia et al. 2013; Tortella et al. 2013a). Briefly, Andisol top soil (Freire serie) was mixed with wheat straw and peat in a volumetric proportion of 1:2:1. The soil was collected from 0 to $15 \mathrm{~cm}$ and sieved $(<3 \mathrm{~mm})$. Wheat straw was cut into $2 \mathrm{~cm}$ pieces using a food processor. Commercial peat, obtained from a local market, contained $33 \%$ cellulose and $21 \%$ lignin. The soil provided $\mathrm{pH} 5.4,18 \mathrm{mg} \mathrm{kg}^{-1}$ of available nitrogen, $17 \mathrm{mg} \mathrm{kg}^{-1}$ of available phosphorus and $12 \%$ organic matter.

Wheat straw provided $\mathrm{pH} 5.9,0.56 \%$ total nitrogen, $9.9 \%$ lignin, $41 \%$ cellulose and $50 \%$ organic matter. The biomixture was stored in a polypropylene bag at $4^{\circ} \mathrm{C}$ until use. The final biomixture composition provided $\mathrm{pH} 4.8,0.54 \%$ total nitrogen and $30 \%$ organic carbon, a with $\mathrm{C} / \mathrm{N}$ ratio of 57 . In order to obtain a homogeneous biomixture, the constituents were vigorously mixed and then moistened with distilled water to $60 \%$ water-holding capacity (WHC). The biomixture was incubated for 30 days at $20 \pm 1{ }^{\circ} \mathrm{C}$ in a closed polypropylene bag before use.

In order to establish a biobed system, the biomixture $(1000 \mathrm{~g})$ was transferred to $5000 \mathrm{~mL}$ glass pots and inoculated with $10 \%\left(\mathrm{w} \mathrm{w}^{-1}\right)$ immobilized fungi on PS. Fungal inoculum was prepared by incorporating immobilized fungi on PS at a depth of $5 \mathrm{~cm}$ below the biobed surface. Thereafter, $80 \mathrm{mg} \mathrm{kg}^{-1}$ atrazine was spread over the biobed.

Four biobed systems were prepared: biomixture + atrazine, biomixture + fungal inoculum, biomixture + atrazine + fungal inoculum and biomixture as control. Each experiment was carried out in triplicate using a destructive sampling mode.

\section{Residual atrazine and their metabolites}

Atrazine was purchased from Bayer Chemicals; deethylatrazine (DEA) and deisopropylatrazine (DIA) were purchased from Aldrich; all other chemicals were purchased from Merck Chile. In order to extract atrazine from the biobed, $20 \mathrm{~mL}$ of methanol was added to $10 \mathrm{~g}$ samples and incubated for $1 \mathrm{~h}$ at $25^{\circ} \mathrm{C}$ under shaking (350 rpm). Afterwards, samples were sonicated at full power for $30 \mathrm{~min}$, subsequently centrifuged at 10,000 rpm for $10 \mathrm{~min}$ and filtered through $0.2 \mu \mathrm{m}$ PTFS membrane filters (Whatman). Residual atrazine was extracted from the biobed system 15 days after initial application. This extraction process was performed twice for each sample and expressed in $\mathrm{mg} \mathrm{kg}^{-1}$. Atrazine concentration in the final supernatant was measured by high-performance liquid chromatography (HPLC, VWR Hitachi). Samples $(20 \mu \mathrm{L})$ were injected into a Rheodyne 7725 injector supplied with a Merck Hitachi L-7100 pump, in an apparatus equipped with a Merck Hitachi L-7455 diode array detector set at $290 \mathrm{~nm}$.

Separation was performed using a C18 column. The mobile phase was $100 \%$ methanol and the flow rate was set at $1 \mathrm{~mL} \mathrm{~min}{ }^{-1}$. The recovery rate of atrazine was greater than $85 \%$. The half-life value of atrazine was obtained using the first-order kinetic equation as concentration $=C_{0} e^{-k t}$, and from this equation Equation (1) was obtained:

$$
T 1 / 2=\operatorname{Ln}(2) / k
$$

where $k$ is the first-order rate constant $\left(d^{-1}\right)$.

\section{Microbial activity in the biobed system}

Phenoloxidase (PO) activity was determined every 15 days and was performed using 3-methyl-2benzothiazolinone hydrazone (MBTH) and 3-(dimethylamino) benzoic acid (DMAB) (Castillo et al. 1994). Because no individual activity was determined for MnP, LiP and Lcc, PO activity represents the sum of all three. Briefly, biobed samples $(10 \mathrm{~g})$ were shaken $(150 \mathrm{rpm})$ for $2 \mathrm{~h}$ with $25 \mathrm{~mL} 100 \mathrm{mM}$ succinate-lactate buffer ( $\mathrm{pH} \mathrm{4.5)}$ and then centrifuged at $4000 \mathrm{rpm}$ for $20 \mathrm{~min}$. The supernatant was collected, filtered through a $0.45 \mu \mathrm{m}$ membrane and PO activity was 
immediately determined by spectrophotometry (Spectronic Genesis 2PC) at $590 \mathrm{~nm}$ ( $\varepsilon=0.053 \mu \mathrm{M}^{-1} \mathrm{~cm}^{-1}$ ), adding $10 \mu \mathrm{L}$ of $10 \mathrm{mM} \mathrm{H}_{2} \mathrm{O}_{2}$ to the reaction mixture, which then contained $300 \mu \mathrm{L}$ of $6.6 \mathrm{mM} \mathrm{DMAB}, 100 \mu \mathrm{L}$ of $1.4 \mathrm{mM} \mathrm{MBTH}, 30 \mu \mathrm{L}$ of $20 \mathrm{mM} \mathrm{MnSO} \mathrm{m}_{4}$ and $1560 \mu \mathrm{L}$ of the filtered sample.

Fluorescein diacetate (FDA): was determined in the biobed system (Schnürer \& Rosswall 1982). Briefly, $1 \mathrm{~g}$ of incubated biobed material was transferred to a flask containing $9.9 \mathrm{~mL} 0.1 \mathrm{mM}$ sodium phosphate buffer $(\mathrm{pH} 7.8)$ and FDA solution $\left(2.0 \mathrm{mg} \mathrm{mL}^{-1}\right)$. After $1 \mathrm{~h}$ incubation at $25^{\circ} \mathrm{C}$, the reaction was stopped with $10 \mathrm{~mL}$ acetone and colorimetric measurements were performed at $490 \mathrm{~nm}$. Results were plotted against a calibration curve with standard quantities of FDA and expressed in $\mu \mathrm{g}$ FDA g ${ }^{-1} \mathrm{~h}^{-1}$.

Respiration activity was measured as $\mathrm{CO}_{2}$ produced and absorbed in a $0.2 \mathrm{M} \mathrm{NaOH}$ solution at $20^{\circ} \mathrm{C}$, and was plotted against time. The values are expressed in $\mathrm{mg} \mathrm{CO}_{2} \mathrm{~g}^{-1}$ dry biobed (lannotti et al. 1994).

All experiments were conducted using three independent replicates and statistical analysis was performed. Data were subjected to a multi-way analysis of variance and the averages were compared by Tukey's range tests.

\section{Results and discussion}

\section{Fungal immobilization and inoculation}

In the present study, an evaluation of fungal immobilization over 20 days on PS was performed. An assay based on SEM microscopy was done. Overall, all fungal strains evaluated were efficient in colonizing both surface and core of the PS and no differences in colonization were observed over the assay. Uniform fungal growth across the interspaces of the PS was observed. The behavior of $T$. versicolor HL01 at both surface and core of the lignocellulosic support can be observed on the SEM photomicrographs in Figure 1. A similar growth pattern was observed for S. hirsutum Ru-104 and

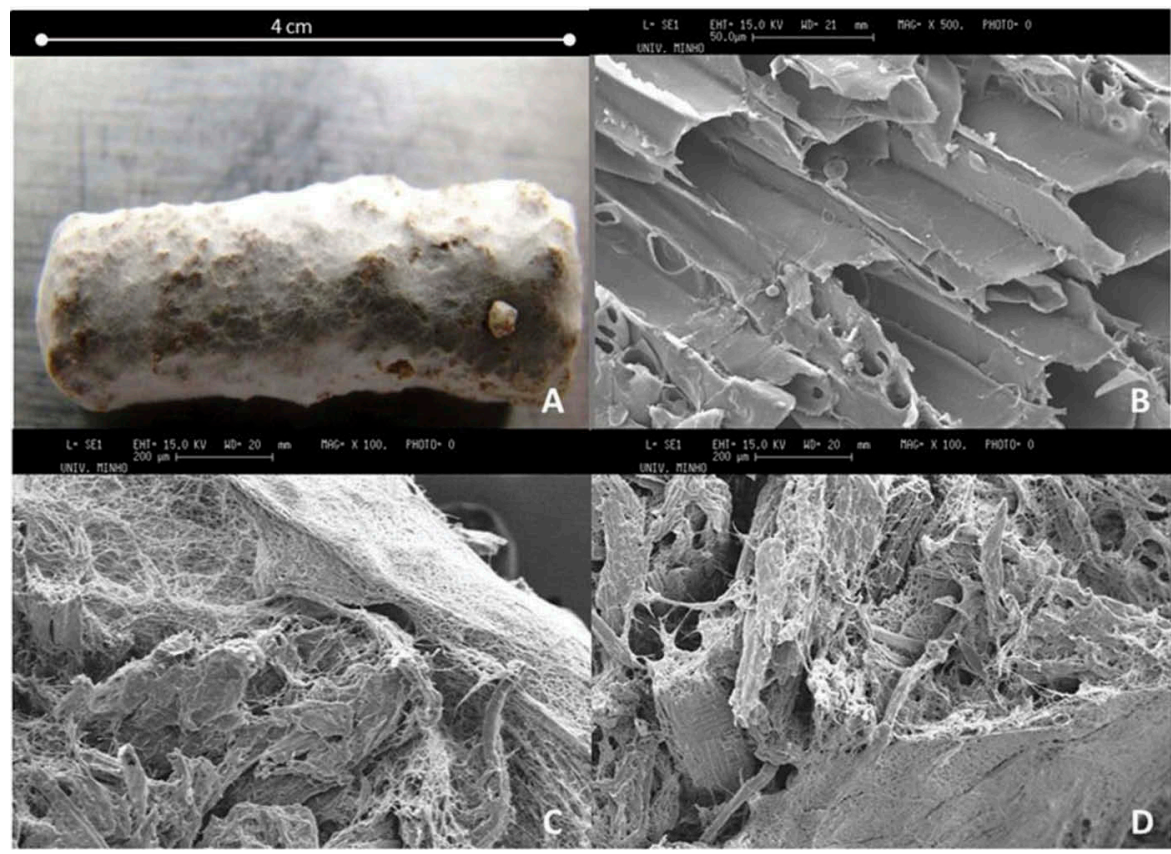

Figure 1. Images of (A) T. versicolor on PS; and microphotographs obtained by SEM for (B) pure PS core; (C) T. versicolor on PS surface; (D) T. versicolor into PS core. All analysis were made with T. versicolor (HL01) after 7 days of incubation at $30^{\circ} \mathrm{C}$. Bars $A$ $4 \mathrm{~cm}$; B $50 \mu \mathrm{m} ; \mathrm{C}-\mathrm{D} 200 \mu \mathrm{m}$. 
Inonotus sp. SP2 (data not shown). Fungal immobilization facilitated hyphal penetration into the biobed, so that fungi reached contaminants in ways that other non-immobilized microorganisms cannot do. As result, increased fungal dissipation capacity in the biobed surrounding environment was achieved.

Results obtained in this present work are corroborated by previous studies published elsewhere (Walter et al. 2004; Schmidt et al. 2005). In order to evaluate the potential of native white-rot fungi to biodegrade pentachlorophenol, Walter et al. (2004) assessed different species of these microorganisms and observed that $T$. versicolor was the most efficient fungal species with the highest release of chloride on wheat straw support. In addition, Schmidt et al. (2005) evaluated the effect of fungal inoculum properties on the colonization of unsterile soil by three isolates of $T$. versicolor. The authors assessed fungal inoculum concentration and its age, from 3 to 21 days, and observed that colonization of soil by $T$. versicolor was improved by increasing the corn content of the fungal inoculum. Furthermore, the authors found that younger ( $<7$ days) fungal inocula resulted in better soil colonization. As attested above, in the present work no differences in fungal growth over the process were observed among the three fungal species evaluated.

Biomixture composition can markedly affect biobed efficiency. Typically, biomixture consists of peat, straw and topsoil. Straw guarantees a continuous supply of nutrients and high microbial activity (Karanasios et al. 2010). However, the microbial adaptation step is crucial for a successful bioprocess. Different adaptation systems have been developed worldwide. The microbial immobilization conditions for biomass growth and an additional supply of carbon sources improved microbial performance in a long-term trial (Ottoni et al. 2014).

In order to increase pesticide dissipation, and to protect fungi from native microflora and overcome the low nutrient rate in soil, immobilized white-rot fungi have been used worldwide as live microorganisms combined with carrier supports (Baldrian 2008).

\section{Atrazine dissipation}

The atrazine dissipation rate in the inoculated biobed system was higher than that observed for the non-inoculated example after 60 days (Figure 2A). The highest dissipation rate (93\%) was observed for S. hirsutum Ru-104 after 60 days of incubation. In contrast, the lowest dissipation rate (78\%) was observed for the non-inoculated biobed over an identical period. The metabolites DIA and DEA (Figure $2 \mathrm{~B}$ and $\mathrm{C}$ ) were found during the experiment in different amounts. The low concentration of both metabolites in the biobed system can be explained by the rapid degradation of atrazine in the experiment. The addition of lignocellulosic substrates in the formulation of the biobed can modify atrazine dissipation (Urrutia et al. 2013).

Bending et al. (2002) evaluated the capacity of nine species of white-rot fungi in the degradation of contrasting mono-aromatic pesticides. Degradation of atrazine $\left(20 \mathrm{\mu g} \mathrm{g}^{-1}\right)$ in a sterile and inoculated biomixture composed of soil, wheat straw and peat was assessed. These authors observed a degradation rate of over than $70 \%$ when the biobed was inoculated with Coriolus versicolor or Hypholoma fasciculare. However, no data for atrazine degradation on sterile biobed were presented in their work.

The capacity of $T$. versicolor to actively degrade atrazine $\left(0.5 \mu \mathrm{g} \mathrm{g}^{-1}\right)$ in a non-inoculated calcareous clay soil for up to 24 weeks $\left(20^{\circ} \mathrm{C}\right)$ was tested (Bastos \& Magan 2009). The authors claimed that fungal inoculation increased atrazine degradation up to $98 \%$ and $85 \%$ at -0.7 and $-2.8 \mathrm{MPa}$, respectively. These data are close to the $88.8 \%$ obtained in the present study for $T$. versicolor HL01. For soil with no fungal presence and under the same pressure conditions, atrazine degradation was respectively 96 and 50\%, and in our case without inoculant $78 \%$.

According to our results, atrazine had a lower half-life $\left(t_{1 / 2}\right)$ in the inoculated biobed than in the non-inoculated (Figure 2). Among the species evaluated, fungi with the highest capacity to degrade atrazine over the time are ranked in the following increasing order of $t_{1 / 2}: S$. hirsutum $\mathrm{Ru}-104=16$ days, T. versicolor HL01 $=21$ days, and Inonotus sp. $=26$ days. S. hirsutum Ru-104 

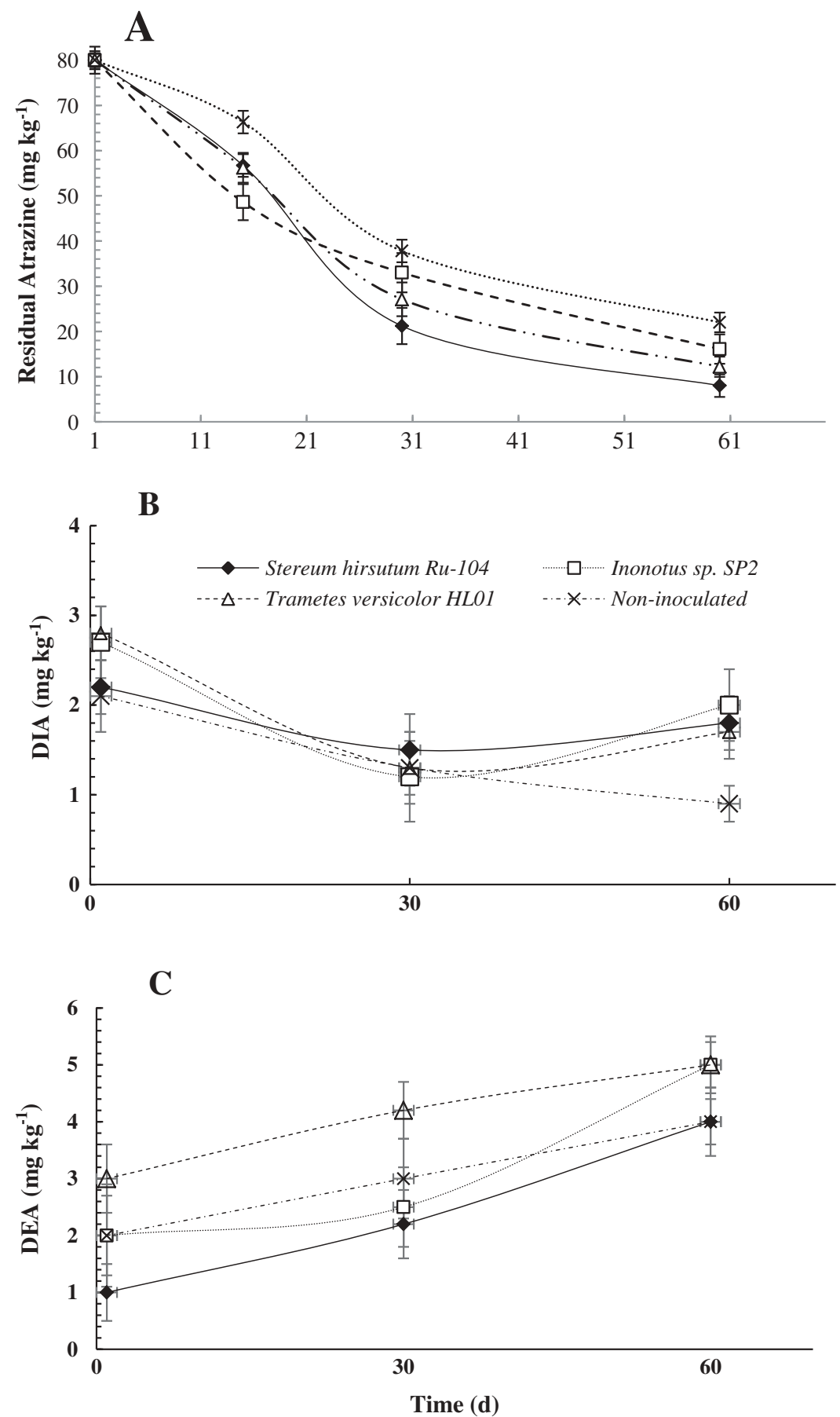

Figure 2. Residual atrazine (A) and its metabolites (B) and (C) after 60 days at $20^{\circ} \mathrm{C}$, in a biomixture inoculated with immobilized white-rot fungi.

demonstrated optimal dissipation performance. Moreover, for the non-inoculated biobed the $t_{1 / 2}$ was 30 days. The results obtained here are in agreement with data previously reported by Urrutia et al. (2013), who where studied atrazine dissipation (100 $\mathrm{mg} \mathrm{kg}^{-1}$ ) in a non-inoculated biobed composed of straw, topsoil and peat. $\mathrm{A} t_{1 / 2}$ of 28 days for atrazine was found. Furthermore, Tortella 
et al. (2013b) observed that terpenes added individually at relatively low concentrations $\left(50 \mu \mathrm{g} \mathrm{kg}^{-1}\right)$ significantly enhanced both atrazine dissipation and biological activity at the beginning of incubation. According to these authors, successive terpene applications were seen as a way of sustaining microbial activity and improving atrazine dissipation in the long term. However, this practice proved not to be not cost effective but gives new insights into exploring the importance of different organic substrates in biobed systems, to increase microbial activity for pesticide dissipation and further decrease ( $<16$ days) atrazine $t_{1 / 2}$.

An attractive alternative to reducing atrazine $t_{1 / 2}$ could be the use of natural residues with high terpene content, such as orange peel and eucalyptus leaves, which would be cost effective. Furthermore, the use of natural residues as a supplement or support for fungal growth and enzyme production has been described as a promising process at the industrial level (Maciel et al. 2013, 2014). In fact, as described above, the evidence suggests that a complex of extracellular enzymes is responsible at least in part for the degradative capabilities of the fungal species assessed (Ottoni et al. 2014).

\section{Biological activity in the biomixture}

After inoculation of immobilized white-rot fungi isolates, PO activity in the biobeds contaminated with atrazine was assessed. Results obtained for the non-inoculated biobed indicate PO activity of around $0.1-0.2 \mathrm{U} \mathrm{kg}^{-1}$. In contrast, for the inoculated biobed, PO activity varied from 0.1 to $0.7 \mathrm{U} \mathrm{kg}^{-1}$. Overall, enzymatic activity increased over time reaching a maximum at the 30th day, after which it decreased to the 60th day of assay (Figure 3).

Among the fungal species evaluated, the optimal enzyme producer was T. versicolor HL01, which resulted in PO activity of $0.7 \mathrm{U} \mathrm{kg}^{-1}$ at the 30th day of assay; S. hirsutum Ru-104 followed T. versicolor HL01 in terms of enzymatic activity. This fungal isolate presented its optimal PO activity at the 15 th day of assay $\left(0.4 \mathrm{U} \mathrm{kg}^{-1}\right)$. In contrast, Inonotus sp. SP2 showed a PO activity comparable to that observed for the non-inoculated biobed (Figure 3).

In the present work, decreased PO activity in the biobed over time may be explained by reduced phenolic compound availability. In addition, microbial cells are able to produce intracellular and extracellular PO for a variety of purposes. Excretion or cell lysis allows these enzymes to enter the environment where their aggregate activity mediates the key ecosystem functions of lignin degradation, humification, carbon mineralization and dissolved organic carbon export (Sinsabaugh 2010).

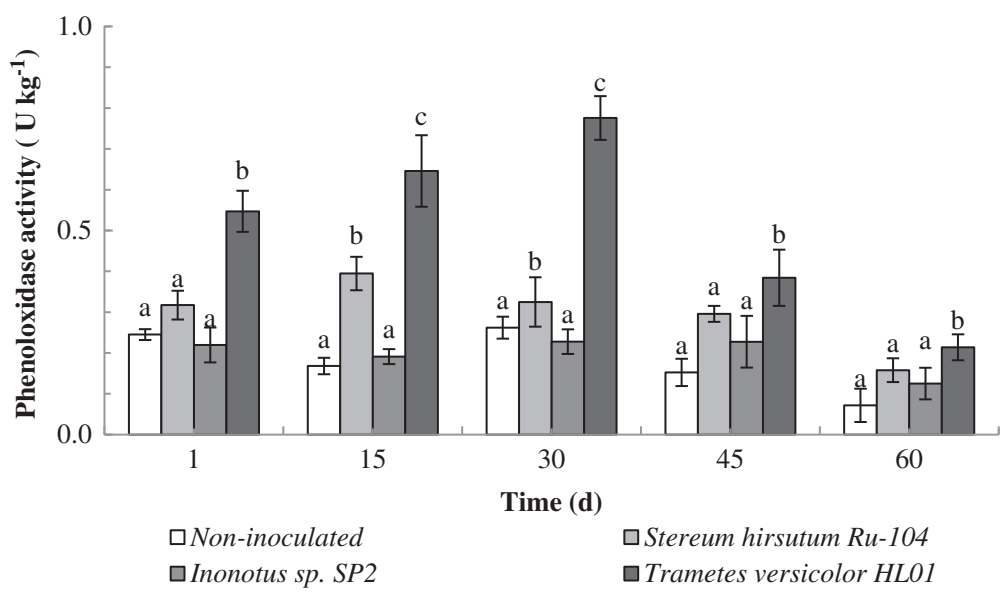

Figure 3. Phenoloxidase activity after 60 days at $20^{\circ} \mathrm{C}$, in a biomixture inoculated with immobilized white-rot fungi. Upper-case letters indicate significance differences $(P \leq 0.05)$ among immobilized white-rot fungi. 
Many researchers have assumed that degradation of pesticides by white-rot fungi is mediated by peroxidases, enzymes involved in the degradation of lignin. In the present study, the biobed tested included $50 \%$ wheat straw. Furthermore, PS contains carbon sources readily available and other compounds related to lignin degradation. Together, this could explain PO activity in the biobed system tested (Tortella et al. 2013a).

FDA activity was used as an estimation of biological potential in the complex mixture composing the biobed. This provides a measurement of microbial biomass by determining a spectrum of microbial enzyme activity (lipases, proteases, esterases) (Leštan et al. 1996). In all biomixture assays, FDA activity was in the range $30-55 \mu \mathrm{g} \mathrm{g}^{-1} \mathrm{~h}^{-1}$ over the 60 days of incubation (Figure 4). These findings indicate that both the inoculated and non-inoculated biobed were biologically active over the incubation period.

Among all inoculated biobed assays, that with $T$. versicolor HL01 showed the highest FDA activity $\left(53 \mu \mathrm{g} \mathrm{g}^{-} 1 \mathrm{~h}^{-1}\right)$ at the 45 th day. The biobed inoculated with Inonotus sp. SP2 followed $T$. versicolor HLO1 in terms of FDA activity $\left(51 \mathrm{\mu g} \mathrm{g}^{-1} \mathrm{~h}^{-1}\right)$ at the 45th day (Figure 4). In contrast, the non-inoculated biobed showed lower FDA activity over the assay (31-34 $\mathrm{g} \mathrm{g} \mathrm{g}^{-} 1 \mathrm{~h}^{-1}$ ). This result demonstrates that inoculation increases the metabolic activity of microorganisms in a biobed. At the 45th day of assay, FDA activity in the inoculated biobed with S. hirsutum Ru-104, Inonotus sp. SP2 or T. versicolor HL01 showed significant differences $(P \leq 0.05)$ compared with the noninoculated example. Moreover, at the 60th day FDA activity in all biobed assays decreased for values of $30-40 \mathrm{Mg} \mathrm{g}^{-1} \mathrm{~h}^{-1}$.

High values of FDA activity in a non-inoculated biobed contaminated with atrazine were also observed by Urrutia et al. (2013). For these authors this indicated that the high FDA hydrolysis value is indicative that all biobeds were biologically active over the incubation period, and that nonsignificant negative effects were caused by the application of pesticide. In such a non-inoculated system, high biological activity is not always associated with pesticide dissipation. The microorganisms naturally present in the biobed components may not have the capacity to degrade pesticides. In contrast, pesticide dissipation can be greatly improved through the inoculation of specific microorganisms. Moreover, an adaptation or immobilization step can improve microbial efficiency still further.

In the present study, respiratory activity was used as a microbial activity indicator. This activity was higher in the inoculated biobed than in the non-inoculated (Figure 5). A high respiration rate (19 mg CO $2 \mathrm{~g}^{-1} \mathrm{~d}^{-1}$ ) was observed in the biobed inoculated with S. hirsutum Ru-104, followed by that with Inonotus sp. SP2 (17 mg CO $2 \mathrm{~g}^{-1} \mathrm{~d}^{-1}$ ). The high respiration rate in the biobed inoculated with S. hirsutum Ru-104 can be related to the high level of atrazine dissipation in this biobed; it can

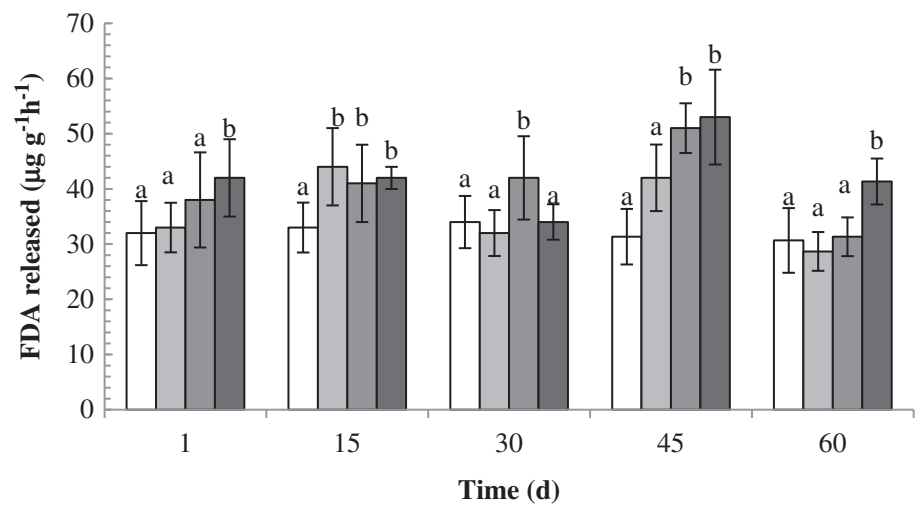

$\square$ Non-inoculated

$\square$ Stereum hirsutum Ru-104

口Inonotus sp. SP2

$\square$ Trametes versicolor HLO1

Figure 4. FDA activity after 60 days at $20^{\circ} \mathrm{C}$, in a biomixture inoculated with immobilized white-rot fungi. Upper-case letters indicate significance difference $(P \leq 0.05)$ among immobilized white-rot fungi. 


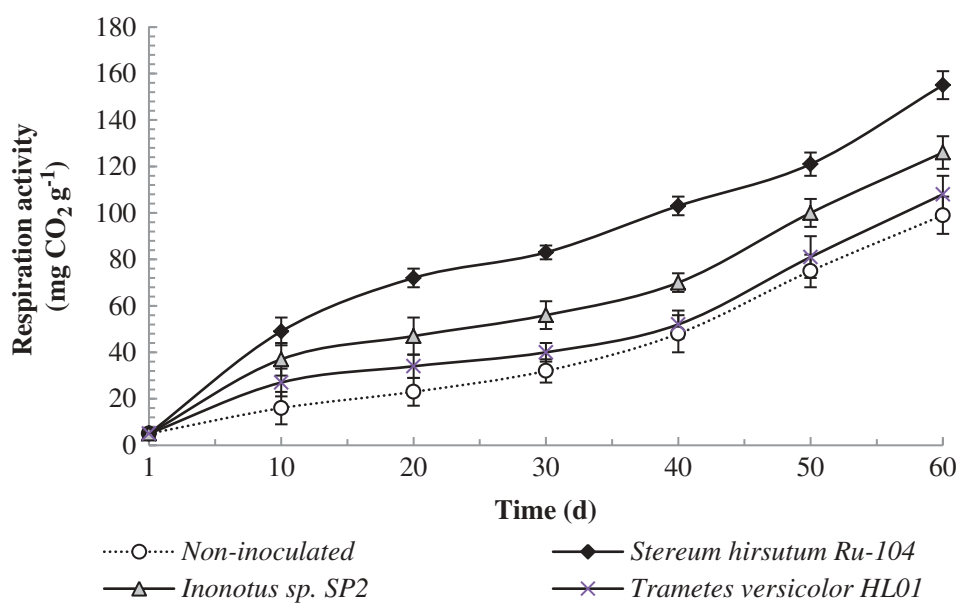

Figure 5. Cumulative respiration activity after 60 days at $20^{\circ} \mathrm{C}$, in a biomixture inoculated with immobilized white-rot fungi. Each value is the mean of three replicates, and the error bars show the standard deviation of the mean.

also be attributed to the presence of an easily degradable carbon source in that environment. In contrast, low respiration rates were observed for the non-inoculated biobed $\left(16 \mathrm{mg} \mathrm{CO} \mathrm{Cg}^{-1} \mathrm{~d}^{-1}\right.$ ) (Figure 5).

The highest respiration activity $\left(155 \mathrm{mg} \mathrm{CO}_{2} \mathrm{~g}^{-1}\right)$ was observed for the biobed inoculated with $S$. hirsutum RU-104 over 60 days of assay. In contrast, the lowest was observed for the non-inoculated biobed $\left(99 \mathrm{mg} \mathrm{CO}_{2} \mathrm{~g}^{-1}\right.$ ). Respiration rates of 126 and $108 \mathrm{mg} \mathrm{CO}_{2} \mathrm{~g}^{-1}$ were observed for the biobeds inoculated with Inonotus sp. SP2 and T. versicolor Ru-104, respectively. Similar results were observed by Tortella et al. (2013b) for a non-inoculated biobed contaminated with atrazine $\left(100 \mathrm{mg} \mathrm{kg}^{-1}\right)$. In this case, the authors replaced wheat straw by barley husk and sawdust in the biomixture. As a result, accumulated respiration after 120 days of incubation was 47 and $42 \mathrm{mg}$ $\mathrm{CO}_{2} \mathrm{~g}^{-1}$, respectively.

Bastos and Magan (2009) observed that respiration was significantly enhanced in soil containing inoculant, particularly in the presence of atrazine, indicating that it remained metabolically active throughout the study. By comparing the data obtained in the present work to those available on the literature, there is clear evidence that inoculation plays an important role in respiratory activity. Furthermore, the inoculation of fungi in PS systems might be a coadjutant in increasing fungal efficiency in enzyme production.

\section{Conclusions}

All fungal strains evaluated were efficient in colonizing both surface and core of the PS, and no differences in the colonization were observed over the assay. Uniform fungal growth across the interspaces of the PS was observed. Moreover, the biobed inoculated with white-rot fungi immobilized on PS resulted in increased atrazine dissipation. Inoculated biobed systems showed higher phenoloxidase activity over time, reaching a maximum at the 30th day after which this decreased to the 60th day of assay. T. versicolor HL01 showed the highest phenoloxidase activity.

FDA activity indicates that both inoculated and non-inoculated biobeds were biologically active over the incubation period. A high respiration rate was observed in the biobed system inoculated with S. hirsutum Ru-104, followed by that inoculated with Inonotus Sp. SP2. This may be related to the high level of atrazine degradation in the latter biobed system. Moreover, it can also be attributed to the presence of an easily degradable carbon source in that environment. In contrast, low respiration rates were observed in the non-inoculated biobed system. 


\section{Disclosure statement}

No potential conflict of interest was reported by the authors.

\section{Funding}

This work was supported by Postdoctoral Scholarship, Universidad de La Frontera [UFRO-2015-2016] and FONDECYT [1120963], and CONICYT FONDAP [15130015].

\section{References}

Baldrian P. 2008. Wood-inhabiting ligninolytic basidiomycetes in soils: ecology and constraints for applicability in bioremediation. Fungal Ecol. 1:4-12.

Bastos AC, Magan N. 2009. Trametes versicolor: potential for atrazine bioremediation in calcareous clay soil, under low water availability conditions. Int Biodeterior Biodegradation. 63:389-394.

Bending G, Friloux M, Walker A. 2002. Degradation of contrasting pesticides by white rot fungi and its relationship with ligninolytic potential. FEMS Microbiol Lett. 212:59-63.

Castillo MD, Stenstrom J, Ander P. 1994. Determination of manganese peroxidase activity with 3-methyl-2-benzothiazolinone hydrazone and 3-(dimethylamino)benzoic acid. Anal Biochem. 218:399-404.

Castillo MDP, Torstensson L, Stenström J. 2008. Biobeds for environmental protection from pesticide use: a review. J Agric Food Chem. 56:6206-6219.

Castillo M, Von Wirén-Lehr S, Scheunert I, Torstensson L. 2001. Degradation of isoproturon by the white-rot fungus Phanerochaete chrysosporium. Biol Fertil Soils. 33:521-528.

D'Annibale A, Rosetto F, Leonardi V, Federici F, Petruccioli M. 2006. Role of autochthonous filamentous fungi in bioremediation of a soil historically contaminated with aromatic hydrocarbons. Appl Environ Microbiol. 72:28-36.

Iannotti DA, Grebus ME, Toth BL, Madden LV, Hoitink HA. 1994. Oxygen respirometry to assess stability and maturity of composted municipal solid waste. J Environ Qual. 23:1177-1183.

Karanasios E, Tsiropoulos NG, Karpouzas DG, Menkissoglu-Spiroudi U. 2010. Novel biomixtures based on local mediterranean lignocellulosic materials: evaluation for use in biobed systems. Chemosphere. 80:914-921.

Leštan D, Leštan M, Chapelle JA, Lamar RT. 1996. Biological potential of fungal inocula for bioaugmentation of contaminated soils. J Ind Microbiol. 16:286-294.

Maciel M, Ottoni C, Herculano PN, Porto T, Porto A, Santos C, Lima N, Moreira KA, Souza-Motta C. 2014. Purification of polygalacturonases produced by Aspergillus niger using an aqueous two-phase system. Fluid Phase Equilib. 371:125-130.

Maciel M, Ottoni C, Santos C, Lima N, Moreira K, Souza-Motta C. 2013. Production of polygalacturonases by Aspergillus section nigri strains in a fixed bed reactor. Molecules. 18:1660-1671.

Mougin C, Pericaud C, Dubroca J, Asther M. 1997. Enhanced mineralization of lindane in soils supplemented with the white-rot basidiomycete Phanerochaete chrysosporium. Soil Biol Biochem. 29:1321-1324.

Ottoni CA, Lima L, Santos C, Lima N. 2014. Effect of different carbon sources on decolourisation of an industrial textile dye under alkaline-saline conditions. Curr Microbiol. 68:53-58.

Ralebitso TK, Senior E, Verseveld HWV. 2002. Microbial aspects of atrazine degradation in natural environments. Biodegradation. 13:11-19.

Rodríguez-Rodríguez CE, Castro-Gutiérrez V, Chin-Pampillo JS, Ruiz-Hidalgo K. 2013. On-farm biopurification systems: role of white rot fungi in depuration of pesticide-containing wastewaters. FEMS Microbiol Lett. 345:1-12.

Rubilar O, Tortella G, Cea M, Acevedo F, Bustamante M, Gianfreda L, Diez MC. 2011. Bioremediation of a Chilean Andisol contaminated with pentachlorophenol (PCP) by solid substrate cultures of white-rot fungi. Biodegradation. 22:31-41.

Schmidt KR, Chand S, Gostomski PA, Boyd-Wilson KSH, Ford C, Walter M. 2005. Fungal inoculum properties and its effect on growth and enzyme activity of Trametes versicolor in soil. Biotechnol Prog. 21:377-385.

Schnürer J, Rosswall T. 1982. Fluorescein diacetate hydrolysis as a measure of total microbial activity in soil and litter. Appl Environ Microbiol. 43:1256-1261.

Sinsabaugh RL. 2010. Phenol oxidase, peroxidase and organic matter dynamics of soil. Soil Biol Biochem. 42:391-404.

Tortella G, Mella-Herrera RA, Souza DZ, Rubilar O, Acuña J, Briceño G, Diez MC. 2013a. Atrazine dissipation and its impact on the microbial communities and community level physiological profiles in a microcosm simulating the biomixture of on-farm biopurification system. J Hazard Mater. 260:459-467.

Tortella G, Rubilar O, Gianfreda L, Valenzuela E, Diez MC. 2008. Enzymatic characterization of Chilean native woodrotting fungi for potential use in the bioremediation of polluted environments with chlorophenols. World J Microbiol Biotechnol. 24:2805-2818.

Tortella G, Rubilar O, Stenstrom J, Cea M, Briceño G, Quiroz A, Diez MC, Parra L. 2013b. Using volatile organic compounds to enhance atrazine biodegradation in a biobed system. Biodegradation. 24:711-720. 
Urrutia C, Rubilar O, Tortella G, Diez MC. 2013. Degradation of pesticide mixture on modified matrix of a biopurification system with alternatives lignocellulosic wastes. Chemosphere. 92:1361-1366.

Walter M, Boul L, Chong R, Ford C. 2004. Growth substrate selection and biodegradation of PCP by New Zealand white-rot fungi. J Environ Manage. 71:361-369.

Walter M, Boyd-Wilson K, Boul L, Ford C, Mcfadden D, Chong B, Pinfold J. 2005. Field-scale bioremediation of pentachlorophenol by Trametes versicolor. Int Biodeterior Biodegradation. 56:51-57.

Zhang J, Qiao C. 2002. Novel approaches for remediation of pesticide pollutants. Int J Environ Pollut. 18:423-433. 